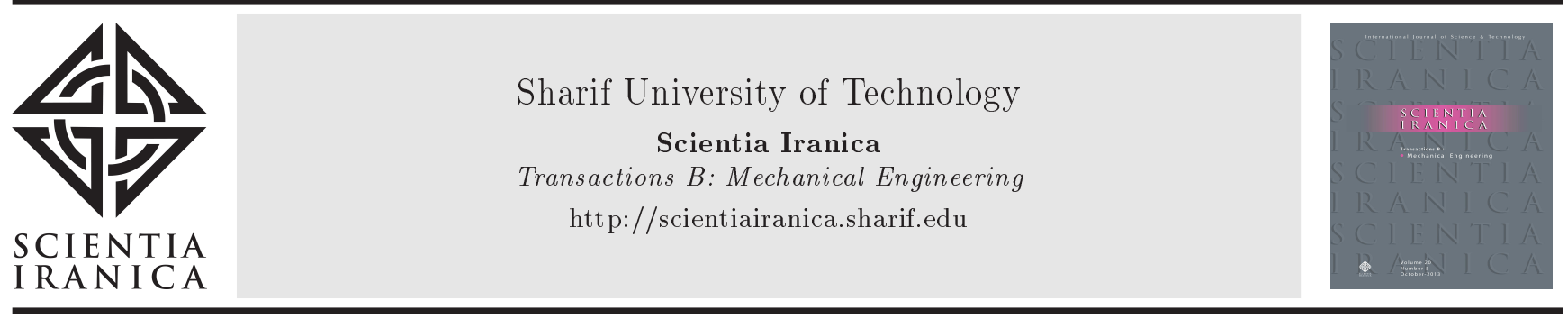

\title{
A new numerical application of the generalized Rosenau-RLW equation
}

\author{
S.B.G. Karakoc* \\ Department of Mathematics, Faculty of Science and Art, Nevsehir Haci Bektas Veli University, Nevsehir, 50300, Turkey.
}

Received 27 March 2018; received in revised form 29 August 2018; accepted 17 November 2018

\section{KEYWORDS}

Generalized Rosenau-

RLW equation;

Finite element

method;

Collocation;

Septic B-spline;

Soliton.

\begin{abstract}
This study implemented a collocation finite element method based on septic B-splines as a tool to obtain the numerical solutions of the nonlinear generalized RosenauRLW equation. One of the advantages of this method is that when the bases are chosen at a high degree, better numerical solutions are obtained. Effectiveness of the method is demonstrated by solving the equation with various initial and boundary conditions. Further, in order to detect the performance of the method, $L_{2}$ and $L_{\infty}$ error norms and two lowest invariants $I_{M}$ and $I_{E}$ were computed. The obtained numerical results were compared with some of those in the literature for similar parameters. This comparison clearly shows that the obtained results are better than and in good conformity with some of the earlier results. Stability analysis demonstrates that the proposed algorithm, based on a Crank Nicolson approximation in time, is unconditionally stable.
\end{abstract}

(C) 2020 Sharif University of Technology. All rights reserved.

\section{Introduction}

This paper is related to the following nonlinear Generalized Rosenau-RLW (GR-RLW) equation:

$$
U_{t}-U_{x x t}+U_{x x x x t}+U_{x}+\left(U^{p}\right)_{x}=0,
$$

with the homogeneous boundary conditions:

$$
\begin{aligned}
& U(a, t)=0, \quad U(b, t)=0, \\
& U_{x}(a, t)=0, \quad U_{x}(b, t)=0, \\
& U_{x x}(a, t)=0, \quad U_{x x}(b, t)=0, \quad t>0,
\end{aligned}
$$

and an initial condition:

\footnotetext{
*. Tel.: +905053919200

E-mail address: sbgkarakoc@nevsehir.edu.tr
}

doi: $10.24200 /$ sci.2018.50490.1721

$$
U(x, 0)=U_{0}(x) \quad a \leq x \leq b,
$$

where $p \geq 2$ is an integer, $U_{x x t}$ is the viscous term, $U_{0}(x)$ is the smooth function, $t$ is time, and $x$ is the space coordinate. The following usual Rosenau-RLW (R-RLW) equation:

$$
U_{t}-U_{x x t}+U_{x x x x t}+U_{x}+2 U U_{x}=0
$$

is obtained by taking $p=2$ in Eq. (1). When $p=3$, the GR-RLW equation is called Modified Rosenau-RLW (MR-RLW) equation. So far, various studies have investigated R-RLW equation. Pan and Zhang [1] examined the usual R-RLW equation by the finite difference method and ideating a conservative algorithm, preserving the original conservative properties for the equation. Pan et al. [2] developed numerical solutions of the R-RLW equation using the finite difference method of Crank-Nicolson type and created the existence of numerical solutions by the 
Brouwer fixed-point theorem. The boundedness and convergence of the approximate solutions for the semidiscrete Galerkin method to the R-RLW equation were analyzed by Atouani and Omrani [3]. Moreover, a Galerkin finite element method was implemented on RRLW equation using cubic B-spline base functions by Yagmurlu et al. [4]. An algorithm to obtain the solution of the nonlinear wave by coupling the Rosenau-KdV and the R-RLW equations was suggested by Wongsaijai and Poochinapan [5]. The numerical solutions of the GR-RLW equation have been studied in recent years. Zuo et al. [6] presented a new conservative difference scheme and showed the existence of its difference-based solutions by Brouwer fixed-point theorem. Numerical solutions for the GR-RLW equation were considered, and an energy conservative linearized finite difference scheme was proposed by Pan and Zhang [7]. Mittal and Jain [8] developed a collocation method for solving the nonlinear GR-RLW equation. A compact finite difference procedure to solve the GR-RLW equation was presented by Wongsaijai et al. [9]. Wang et al. [10, 11] presented a new conservative finite difference scheme for the initial-boundary value problem of the GR-RLW equation and designed a new conservative nonlinear fourth-order compact finite difference algorithm for R-RLW equation given together with the initial and boundary conditions. Arl and Dereli [12] solved the GR-RLW equation by using the meshless kernel-based method of lines. Cai et al. [13] derived the Lagrangians of generalized Rosenau-type equations and suggested the corresponding multi-symplectic formulations.

Nonlinear wave phenomenon is a very important area of scientific research, which many scientists examined in the past. Various mathematical models such as: KdV equation [14-17] (Eq. (5)), RLW equation [18-25] (Eq. (6)), Rosenau equation, and many others have been designed by scientists [5].

$$
\begin{aligned}
& U_{t}+a U U_{x}+b U_{x x x}=0, \\
& U_{t}+U_{x}+a U U_{x}-b U_{x x t}=0 .
\end{aligned}
$$

In the study of the dynamics of dense discrete systems, the case of wave-wave and wave-wall interactions cannot be described using the well-known $\mathrm{KdV}$ equation [26]. Moreover, the slope and the behavior of high amplitude waves may not be well predicted by Eq. (5), since it was modeled under the assumption of weak anharmonicity [26]. To cope with the lack of Eq. (5), the following equation, as proposed by Rosenau $[27,28]$, is used:

$$
U_{t}+U_{x}+U_{x x x x t}+\left(U^{2}\right)_{x}=0 .
$$

Park [29] examined the existence, uniqueness, and regularity of the solutions for the Rosenau equation.
This study designed a septic B-spline collocation method for the GR-RLW equation. The rest of the paper can be summarized briefly as follows: In the second section, the collocation finite element method is applied to the GR-RLW equation. The resulting system can be efficiently solved with a kind of Thomas algorithm. The linear stability analysis of the algorithm is investigated in Section 3. In the fourth section, the motion of the single solitary wave is examined in the case of the problem with different initial and boundary conditions. The numerical results are given both in tables and figures, and the obtained results are also compared with some of those available in the literature. Section 5 concludes this paper with a summary of findings.

\section{Collocation method with septic B-splines}

To carry out numerical computations, the solution area of the problem is limited over an interval $a \leq x \leq$ $b$. Consider the partition of the space interval $[a, \bar{b}]$ into equally sized finite elements of length $h$ at the points $x_{m}$ such that $a=x_{0}<x_{1}<\ldots<x_{N}=b$ and $h=\frac{b-a}{N}$. The set of septic B-spline functions $\left\{\phi_{-3}(x), \phi_{-2}(x), \ldots, \phi_{N+3}(x)\right\}$ forms a basis over the solution region $[a, b]$. The numerical solution $U_{N}(x, t)$ is expressed in terms of the septic B-splines as follows:

$$
U_{N}(x, t)=\sum_{m=-3}^{N+3} \phi_{m}(x) \delta_{m}(t),
$$

where $\delta_{m}(t)$ is the time-dependent parameter and will be defined under the boundary and collocation conditions [30]. Septic B-splines $\phi_{m}(x)(m=-3,-2, \ldots, N+$ $3)$ at the knots $x_{m}$ are designated over the interval $[a, b]$ by Prenter [31] as calculated by Eq. (9) shown in Box I. The septic B-splines are used to overcome higher order derivatives in the equation and, when the bases are chosen at a high degree, better numerical results are generally obtained. A characteristic finite interval $\left[x_{m}, x_{m+1}\right]$ is projected to the interval $[0,1]$ by a local coordinate conversion given by $h \xi=x-x_{m}$, $0 \leq \xi \leq 1$ [32]. Therefore, the septic B-splines (9) are given in terms of $\xi$ over [0,1] as follows:

$$
\begin{aligned}
& \phi_{m-3}= 1-7 \xi+21 \xi^{2}-35 \xi^{3}+35 \xi^{4}-21 \xi^{5} \\
&+7 \xi^{6}-\xi^{7}, \\
& \phi_{m-2}= 120-392 \xi+504 \xi^{2}-280 \xi^{3}+84 \xi^{5} \\
&-42 \xi^{6}+7 \xi^{7}, \\
& \phi_{m-1}= 1191-1715 \xi+315 \xi^{2}+665 \xi^{3}-315 \xi^{4} \\
& \quad-105 \xi^{5}+105 \xi^{6}-21 \xi^{7}, \\
& \phi_{m}=2416-1680 \xi+560 \xi^{4}-140 \xi^{6}+35 \xi^{7},
\end{aligned}
$$




$$
\phi_{m}(x)=\frac{1}{h^{7}} \begin{cases}\left(x-x_{m-4}\right)^{7} & {\left[x_{m-4}, x_{m-3}\right]} \\ \left(x-x_{m-4}\right)^{7}-8\left(x-x_{m-3}\right)^{7} & {\left[x_{m-3}, x_{m-2}\right]} \\ \left(x-x_{m-4}\right)^{7}-8\left(x-x_{m-3}\right)^{7}+28\left(x-x_{m-2}\right)^{7} & {\left[x_{m-2}, x_{m-1}\right]} \\ \left(x-x_{m-4}\right)^{7}-8\left(x-x_{m-3}\right)^{7}+28\left(x-x_{m-2}\right)^{7}-56\left(x-x_{m-1}\right)^{7} & {\left[x_{m-1}, x_{m}\right]} \\ \left(x_{m+4}-x\right)^{7}-8\left(x_{m+3}-x\right)^{7}+28\left(x_{m+2}-x\right)^{7}-56\left(x_{m+1}-x\right)^{7} & {\left[x_{m}, x_{m+1}\right]} \\ \left(x_{m+4}-x\right)^{7}-8\left(x_{m+3}-x\right)^{7}+28\left(x_{m+2}-x\right)^{7} & {\left[x_{m+1}, x_{m+2}\right]} \\ \left(x_{m+4}-x\right)^{7}-8\left(x_{m+3}-x\right)^{7} & {\left[x_{m+2}, x_{m+3}\right]} \\ \left(x_{m+4}-x\right)^{7} & {\left[x_{m+3}, x_{m+4}\right]} \\ 0 & \text { otherwise. }\end{cases}
$$

Box I

$$
\begin{aligned}
\phi_{m+1}= & 1191+1715 \xi+315 \xi^{2}-665 \xi^{3}-315 \xi^{4} \\
& +105 \xi^{5}+105 \xi^{6}-35 \xi^{7} \\
\phi_{m+2}= & 120+392 \xi+504 \xi^{2}+280 \xi^{3}-84 \xi^{5}-42 \xi^{6} \\
& +21 \xi^{7} \\
\phi_{m+3}= & +7 \xi+21 \xi^{2}+35 \xi^{3}+35 \xi^{4}+21 \xi^{5} \\
& +7 \xi^{6}-\xi^{7} \\
\phi_{m+4}= & \xi^{7} .
\end{aligned}
$$

For the problem, the finite elements are described with the space $\left[x_{m}, x_{m+1}\right]$. According to Eqs. (9) and (8), the nodal values of $U_{m}, U_{m}^{\prime}, U_{m}^{\prime \prime}, U_{m}^{\prime \prime \prime}$, and $U_{m}^{i v}$ are given in terms of the element parameters $\delta_{m}$ in [33]:

$$
\begin{aligned}
U_{N}\left(x_{m}, t\right)= & U_{m}=\delta_{m-3}+120 \delta_{m-2}+1191 \delta_{m-1} \\
& +2416 \delta_{m}+1191 \delta_{m+1}+120 \delta_{m+2}+\delta_{m+3}, \\
U_{m}^{\prime}= & \frac{7}{h}\left(-\delta_{m-3}-56 \delta_{m-2}-245 \delta_{m-1}+245 \delta_{m+1}\right. \\
& \left.+56 \delta_{m+2}+\delta_{m+3}\right), \\
U_{m}^{\prime \prime}= & \frac{42}{h^{2}}\left(\delta_{m-3}+24 \delta_{m-2}+15 \delta_{m-1}-80 \delta_{m}\right. \\
+ & \left.15 \delta_{m+1}+24 \delta_{m+2}+\delta_{m+3}\right), \\
U_{m}^{\prime \prime \prime}= & \frac{210}{h^{3}}\left(-\delta_{m-3}-8 \delta_{m-2}+19 \delta_{m-1}-19 \delta_{m+1}\right. \\
+ & \left.8 \delta_{m+2}+\delta_{m+3}\right), \\
U_{m}^{i v}= & \frac{840}{h^{4}}\left(\delta_{m-3}-9 \delta_{m-1}+16 \delta_{m}-9 \delta_{m+1}+\delta_{m+3}\right),
\end{aligned}
$$

and the variation of $U$ over the element $\left[x_{m}, x_{m+1}\right]$ is given as follows [34]:

$$
U=\sum_{m=-3}^{N+3} \phi_{m} \delta_{m}
$$

For Eq. (12), in the solution area of the problem, the first septic B-spline base function's index is -3 , and the last septic B-spline base function's index is $N+3$. When we define the collocation points with the nodes and use Eqs. (11) to utilize $U_{m}$ (its space derivatives) and substitute it into Eq. (1), a set of ordinary differential equations of the form is given [30]:

$$
\begin{aligned}
\dot{\delta}_{m-3} & +120 \dot{\delta}_{m-2}+1191 \dot{\delta}_{m-1}+2416 \dot{\delta}_{m} \\
& +1191 \dot{\delta}_{m+1}+120 \dot{\delta}_{m+2}+\dot{\delta}_{m+3} \\
& +\frac{7}{h}\left(-\delta_{m-3}-56 \delta_{m-2}-245 \delta_{m-1}+245 \delta_{m+1}\right. \\
& \left.+56 \delta_{m+2}+\delta_{m+3}\right)-\frac{42}{h^{2}} \dot{\delta}_{m-3}+24 \dot{\delta}_{m-2} \\
& \left.+15 \dot{\delta}_{m-1}-80 \dot{\delta}_{m}+15 \dot{\delta}_{m+1}+24 \dot{\delta}_{m+2}+\dot{\delta}_{m+3}\right) \\
& +\frac{840}{h^{4}}\left(\dot{\delta}_{m-3}-9 \dot{\delta}_{m-1}+16 \dot{\delta}_{m}-9 \dot{\delta}_{m+1}+\dot{\delta}_{m+3}\right) \\
& +p \frac{7}{h} Z_{m}\left(-\delta_{m-3}-56 \delta_{m-2}-245 \delta_{m-1}\right. \\
& \left.+245 \delta_{m+1}+56 \delta_{m+2}+\delta_{m+3}\right)=0
\end{aligned}
$$

where $Z_{m}=\left(\delta_{m-3}+120 \delta_{m-2}+1191 \delta_{m-1}+2416 \delta_{m}+\right.$ $\left.1191 \delta_{m+1}+120 \delta_{m+2}+\delta_{m+3}\right)^{p-1}[33,35]$.

If parameters $\delta_{i}$ and the time derivatives $\dot{\delta}_{i}$ in Eq. (13) are separated by the Crank-Nicolson formula:

$$
\delta_{i}=\frac{\delta_{i}^{n+1}+\delta_{i}^{n}}{2}
$$

and the usual finite difference approximation:

$$
\dot{\delta}_{i}=\frac{\delta_{i}^{n+1}-\delta_{i}^{n}}{\Delta t}
$$


a repetition relationship is derived between two time levels $n$ and $n+1$ relating to two unknown parameters $\delta_{i}^{n+1}$, and $\delta_{i}^{n}$ for $i=m-3, m-2, \ldots, m+2, m+3$

$$
\begin{aligned}
\gamma_{1} \delta_{m-3}^{n+1} & +\gamma_{2} \delta_{m-2}^{n+1}+\gamma_{3} \delta_{m-1}^{n+1}+\gamma_{4} \delta_{m}^{n+1} \\
& +\gamma_{5} \delta_{m+1}^{n+1}+\gamma_{6} \delta_{m+2}^{n+1}+\gamma_{7} \delta_{m+3}^{n+1} \\
& =\gamma_{7} \delta_{m-3}^{n}+\gamma_{6} \delta_{m-2}^{n}+\gamma_{5} \delta_{m-1}^{n}+ \\
& \gamma_{4} \delta_{m}^{n}+\gamma_{3} \delta_{m+1}^{n}+\gamma_{2} \delta_{m+2}^{n}+\gamma_{1} \delta_{m+3}^{n}
\end{aligned}
$$

where:

$$
\begin{aligned}
& \gamma_{1}=\left[1-E\left(1+p Z_{m}\right)-M+K\right], \\
& \gamma_{2}=\left[120-56 E\left(1+p Z_{m}\right)-24 M\right], \\
& \gamma_{3}=\left[1191-245 E\left(1+p Z_{m}\right)-15 M-9 K\right], \\
& \gamma_{4}=[2416+80 M+16 K], \\
& \gamma_{5}=\left[1191+245 E\left(1+p Z_{m}\right)-15 M-9 K\right], \\
& \gamma_{6}=\left[120+56 E\left(1+p Z_{m}\right)-24 M\right], \\
& \gamma_{7}=\left[1+E\left(1+p Z_{m}\right)-M+K\right], \\
& m=0,1, \ldots, N \quad E=\frac{7}{2 h} \Delta t, \\
& M=\frac{42}{h^{2}}, \quad K=\frac{840}{h^{4}} .
\end{aligned}
$$

System (16) involves $(N+1)$ linear equations containing $(N+7)$ unknown coefficients $\left(\delta_{-3}, \delta_{-2}, \delta_{-1}\right.$, $\left.\ldots, \delta_{N+1}, \delta_{N+2}, \delta_{N+3}\right)^{T}$. Six additional restraints are required to get a unique solution for this system. These are obtained from the boundary conditions (2) and can be used to remove $\delta_{-3}, \delta_{-2}, \delta_{-1}$ and $\delta_{N+1}, \delta_{N+2}, \delta_{N+3}$ from Systems (16), leading to a matrix equation for the $N+1$ unknowns $d^{n}=\left(\delta_{0}, \delta_{1}, \ldots, \delta_{N}\right)^{T}$ of the form [30]:

$$
Y \mathbf{d}^{\mathbf{n}+1}=Z \mathbf{d}^{\mathbf{n}}
$$

The matrices $Y$ and $Z$ are $(N+1) \times(N+1)$ matrices. Two or three inner iterations are implemented on the term $\delta^{n *}=\delta^{n}+\frac{1}{2}\left(\delta^{n}-\delta^{n-1}\right)$ at each time step to overcome the non-linearity caused by $Z_{m}$. Before the beginning of the solution period, initial parameters $d^{0}$ are established by using the initial condition and the following derivatives at the boundaries:

$$
\begin{array}{ll}
U_{N}(x, 0)=U\left(x_{m}, 0\right), & m=0,1,2, \ldots, N \\
\left(U_{N}\right)_{x}(a, 0)=0, & \left(U_{N}\right)_{x}(b, 0)=0, \\
\left(U_{N}\right)_{x x}(a, 0)=0, & \left(U_{N}\right)_{x x}(b, 0)=0, \\
\left(U_{N}\right)_{x x x}(a, 0)=0, & \left(U_{N}\right)_{x x x}(b, 0)=0 .
\end{array}
$$

Therefore, the matrix form of the initial vector $d^{0}$ is given by the expressions shown in Box II [35]

\section{Stability analysis}

To investigate the stability analysis of the presented scheme, it is necessary to use Von-Neumann theory.

$V d^{0}=w$

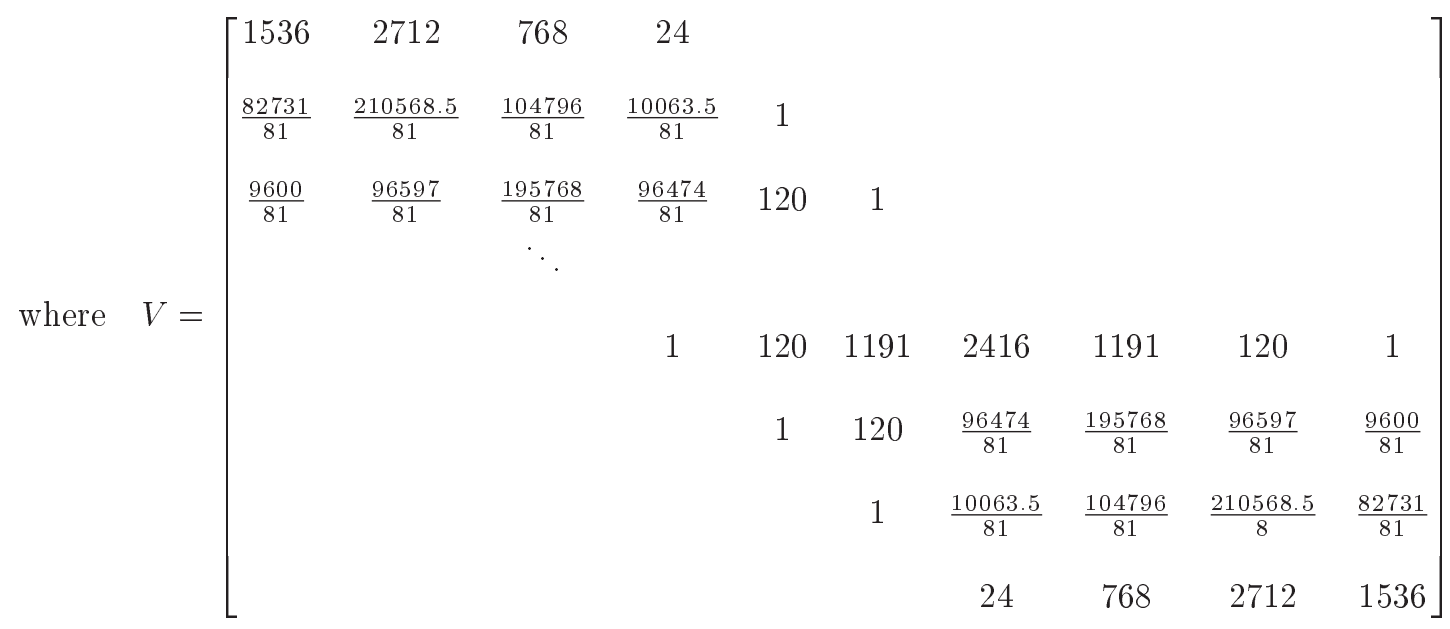

$d^{0}=\left(\delta_{0}, \delta_{1}, \delta_{2}, \ldots, \delta_{N-2}, \delta_{N-1}, \delta_{N}\right)^{T}$ and $w=\left(U\left(x_{0}, 0\right), U\left(x_{1}, 0\right), \ldots, U\left(x_{N-1}, 0\right), U\left(x_{N}, 0\right)\right)^{T}$. 
Assume that the quantity $U^{p}$ in the nonlinear term $U^{p} U_{x}$ is locally constant [30]. Substituting the Fourier mode $\delta_{m}^{n}=\xi^{n} e^{i \sigma m h},(i=\sqrt{-1})$ into Eq. (16), we obtain the following:

$$
\begin{aligned}
\xi^{n+1} & \left(\eta_{1} e^{i(m-3) \theta}+\eta_{2} e^{i(m-2) \theta}+\eta_{3} e^{i(m-1) \theta}\right. \\
& \left.+\eta_{4} e^{i m \theta}+\eta_{5} e^{i(m+1) \theta}+\eta_{6} e^{i(m+2) \theta}+\eta_{7} e^{i(m+3) \theta}\right) \\
& =\xi^{n}\left(\eta_{7} e^{i(m-3) \theta}+\eta_{6} e^{i(m-2) \theta}\right. \\
& +\eta_{5} e^{i(m-1) \theta}+\eta_{4} e^{i m \theta}+\eta_{3} e^{i(m+1) \theta} \\
& \left.+\eta_{2} e^{i(m+2) \theta}+\eta_{1} e^{i(m+3) \theta}\right),
\end{aligned}
$$

where $\sigma$ is the mode number, $h$ is the element size, and $\theta=\sigma h$ :

$$
\begin{aligned}
& \eta_{1}=1-\beta_{1}-\beta_{2}+\beta_{3}, \\
& \eta_{2}=120-56 \beta_{1}-24 \beta_{2}, \\
& \eta_{3}=1191-245 \beta_{1}-15 \beta_{2}-9 \beta_{3}, \\
& \eta_{4}=2416+80 \beta_{2}+16 \beta_{3}, \\
& \eta_{5}=1191+245 \beta_{1}-15 \beta_{2}-9 \beta_{3}, \\
& \eta_{6}=120+56 \beta_{1}-24 \beta_{2}, \\
& \eta_{7}=1+\beta_{1}-\beta_{2}+\beta_{3}, \\
& m=0,1, \ldots, N, \\
& \beta_{1}=\frac{7 \Delta t}{2 h}\left(1+Z_{m}\right), \quad \beta_{2}=\frac{42}{h^{2}}, \quad \beta_{3}=\frac{840}{h^{4}} .
\end{aligned}
$$

If Eq. (19) is simplified the following is obtained:

$$
\xi=\frac{A+i B}{A-i B},
$$

where:

$$
\begin{aligned}
A= & \left(2382-30 \alpha_{2}-18 \alpha_{3}\right) \cos (\theta)+\left(240-48 \alpha_{2}\right) \\
& \cos (2 \theta)+\left(2-2 \alpha_{2}+2 \alpha_{3}\right) \cos (3 \theta) \\
& +\left(2416+80 \alpha_{2}+16 \alpha_{3}\right), \\
B= & \left(490 E\left(1+Z_{m}\right)\right) \sin (\theta)+\left(112 E\left(1+Z_{m}\right)\right) \\
& \sin (2 \theta)+\left(2 E\left(1+Z_{m}\right)\right) \sin (3 \theta) .
\end{aligned}
$$

According to the Fourier stability analysis, for the given scheme to be stable, the condition $|\xi|<1$ must be satisfied. Using the symbolic programming software or using simple calculations, since $a^{2}+b^{2}=$ $a^{2}+(-b)^{2}$, it becomes evident that the modulus of $|\xi|$ is 1 . Consequently the linearized algorithm is found unconditionally stable [36].

\section{Numerical results and discussion}

In this part, in order to verify the correction of our algorithm, some numerical experiments were calculated. For performing the numerical simulation of the motion of single solitary wave for which exact solutions have been given before, four sets of parameters were used and discussed. For the GR-RLW equation, the parameters used by authors $[6,7,9,10]$ to obtain the corresponding results are taken as guiding principles for the current calculations. The initial boundary value problems (1)-(3) have the following conservative quantities:

$$
\begin{aligned}
& I_{M}=\frac{1}{2} \int_{a}^{b} U(x, t) d x, \\
& I_{E}=\frac{1}{2} \int_{a}^{b}\left[U^{2}(x, t)+U_{x}^{2}(x, t)+U_{x x}^{2}(x, t)\right] d x,
\end{aligned}
$$

corresponding to the mass and energy of the shallow water waves, respectively [8]. Susceptibility of the numerical scheme is controlled by both the error norm [37]:

$$
L_{2}=\left\|U^{\text {exact }}-U_{N}\right\|_{2} \simeq \sqrt{h \sum_{J=0}^{N}\left|U_{j}^{\text {exact }}-\left(U_{N}\right)_{j}\right|^{2}},
$$

and the error norm:

$$
L_{\infty}=\left\|U^{\text {exact }}-U_{N}\right\|_{\infty} \simeq \max _{j}\left|U_{j}^{\text {exact }}-\left(U_{N}\right)_{j}\right| .
$$

\subsection{Case 1}

For the first numerical calculation, the parameter $p=$ 2 is chosen with various values of $h$ and $\Delta t$ over the interval $[-30,120]$. For this case, since the exact solution of the problem is [6]:

$$
U(x, t)=\frac{15}{38} \sec h^{4}\left[\frac{\sqrt{13}}{26}\left(x-\frac{169}{133}\right) t\right],
$$

the initial condition for the problem is taken as follows:

$$
U(x, 0)=\frac{15}{38} \sec h^{4}\left[\frac{\sqrt{13}}{26} x\right] .
$$

The invariants and error norms for the single solitary wave over the $-30 \leq x \leq 120$ are shown in Table 1 . Moreover, a comparison of the error norms for different values of $h$ and $\Delta t$ is made, as shown in Table 2 . From Table 2, it is seen that the error norms obtained by the proposed method are found much better than and in good agreement with the others. Behaviors of single soliton time up to $t=40$ are given in Figure 1. According to Figure 1, single soliton travels to the right at an invariable speed and keeps its amplitude and shape with the increasing time, as expected [36]. The amplitude is 0.394736 at $t=0$ and located at $x=0.0$, while it is 0.394623 at $t=60$ and located at $x=76.2$. The certain difference in amplitudes at 
Table 1. The invariants and the error norms for single solitary wave for $p=2$ over the $-30 \leq x \leq 120$.

\begin{tabular}{|c|c|c|c|c|}
\hline$h=\Delta t=0.4$ & $I_{M}$ & $I_{E}$ & $L_{2} \times 10^{-3}$ & $L_{\infty} \times 10^{-3}$ \\
\hline \multicolumn{5}{|l|}{$t$} \\
\hline 0 & 1.8976582 & 0.5331775 & 0 & 0 \\
\hline 20 & 1.8976042 & 0.5331517 & 11.75577 & 4.56558 \\
\hline 40 & 1.8975504 & 0.5331264 & 19.85843 & 7.29166 \\
\hline 60 & 1.8974979 & 0.5331012 & 26.90336 & 9.64250 \\
\hline \multicolumn{5}{|l|}{$h=\Delta t=0.2$} \\
\hline \multicolumn{5}{|l|}{$t$} \\
\hline 0 & 1.8976587 & 0.5331776 & 0 & 0 \\
\hline 20 & 1.8976556 & 0.5331742 & 2.95668 & 1.15327 \\
\hline 40 & 1.8976520 & 0.5331709 & 4.98638 & 1.83689 \\
\hline 60 & 1.8976486 & 0.5331676 & 6.78589 & 2.43822 \\
\hline \multicolumn{5}{|l|}{$h=\Delta t=0.1$} \\
\hline \multicolumn{5}{|l|}{$t$} \\
\hline 0 & 1.8976588 & 0.5331777 & 0 & 0 \\
\hline 20 & 1.8976624 & 0.5331773 & 0.73165 & 0.28627 \\
\hline 40 & 1.8976656 & 0.5331769 & 1.28582 & 0.47045 \\
\hline 60 & 1.8976687 & 0.5331765 & 1.80098 & 0.64362 \\
\hline \multicolumn{5}{|l|}{$h=\Delta t=0.05$} \\
\hline \multicolumn{5}{|l|}{$t$} \\
\hline 0 & 1.8976569 & 0.5331776 & 0 & 0 \\
\hline 20 & 1.8976614 & 0.5331775 & 0.20059 & 0.07495 \\
\hline 40 & 1.8976655 & 0.5331775 & 0.39859 & 0.14198 \\
\hline 60 & 1.8976695 & 0.5331774 & 0.42845 & 0.16500 \\
\hline \multicolumn{5}{|l|}{$h=\Delta t=0.025$} \\
\hline \multicolumn{5}{|l|}{$t$} \\
\hline 0 & 1.8976564 & 0.5331771 & 0 & 0 \\
\hline 20 & 1.8976610 & 0.5331770 & 0.09000 & 0.03165 \\
\hline 40 & 1.8976652 & 0.5331770 & 0.10490 & 0.04479 \\
\hline 60 & 1.8976693 & 0.5331770 & 0.34458 & 0.13007 \\
\hline
\end{tabular}

Table 2. Comparison of error norms for $p=2$.

\begin{tabular}{|c|c|c|c|c|c|c|}
\hline & \multicolumn{3}{|c|}{$L_{2} \times 10^{-2}$} & \multicolumn{3}{|c|}{$L_{\infty} \times 10^{-3}$} \\
\hline$t=40, \quad-60 \leq x \leq 120$ & Present & {$[7]$} & {$[9]$} & Present & {$[7]$} & [9] \\
\hline$h=0.5, \quad \Delta t=0.1$ & 0.125481 & 3.25288 & 0.23029 & 0.46077 & 1.19460 & 0.86284 \\
\hline$h=0.25, \quad \Delta t=0.1$ & 0.125389 & 0.78777 & 0.23608 & 0.46216 & 2.88972 & 0.88670 \\
\hline$t=60, \quad-30 \leq x \leq 120$ & Present & {$[6]$} & {$[10]$} & Present & {$[6]$} & {$[10]$} \\
\hline$h=\Delta t=0.4$ & 2.69033 & 5.47632 & - & 9.64250 & 19.58718 & 3.5235 \\
\hline$h=\Delta t=0.2$ & 0.67858 & 1.38525 & - & 2.43822 & 4.98376 & 0.80413 \\
\hline$h=\Delta t=0.1$ & 0.18009 & 0.34743 & - & 0.64362 & 1.25218 & 0.19123 \\
\hline$h=\Delta t=0.05$ & 0.04284 & 0.086914 & - & 0.16500 & 0.31345 & 0.04659 \\
\hline
\end{tabular}




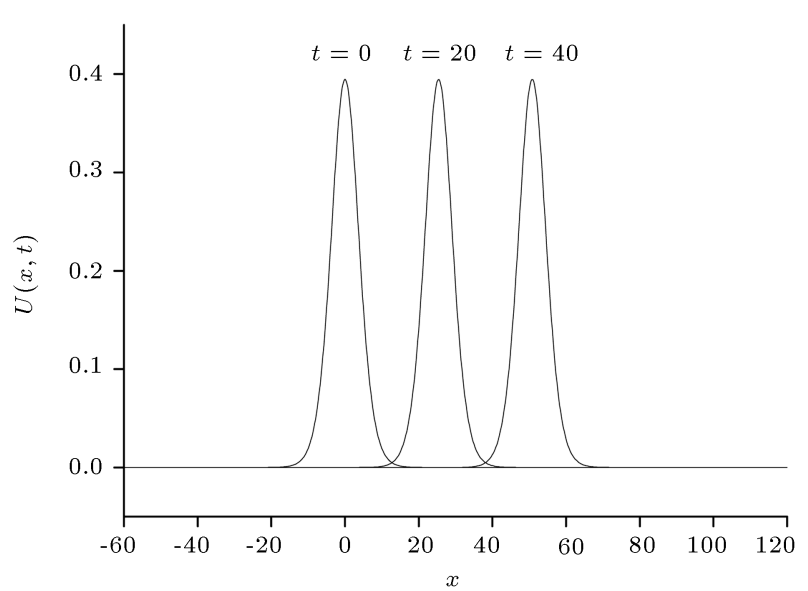

Figure 1. Motion of single solitary wave for $p=2$, $h=0.25$, and $\Delta t=0.1$ over the interval $[-30,120]$ at specified times.

times $t=0$ and $t=40$ is $1.13 \times 10^{-4}$; therefore, there is a minor difference between the amplitudes. Error values between exact and numerical solutions are demonstrated in Figure 2.

\subsection{Case 2}

For the second numerical calculation, the parameter $p=4$ with values of $h=0.25,0.5$ and $\Delta t=0.1$ are used through the interval $[-60,120]$. In this case, the analytical solution of the problem has been found as follows [6]:

$$
U(x, t)=\left(\frac{455}{1482}\right)^{\frac{1}{3}} \sec h^{\frac{4}{3}}\left[\frac{3}{2 \sqrt{29}}\left(x-\frac{841}{741}\right) t\right]
$$

Therefore, the initial condition for this problem is taken as follows:

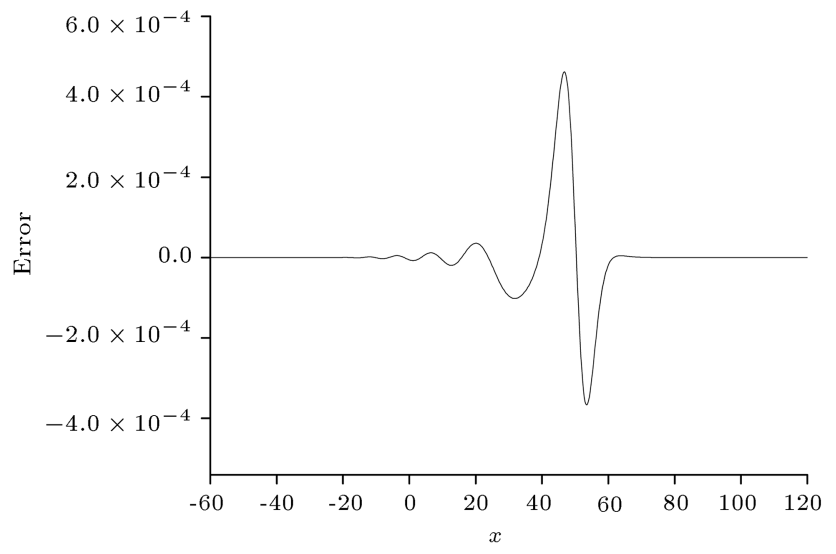

Figure 2. Errors for $p=2, h=0.25$, and $\Delta t=0.1$ at $t=40$.

$$
U(x, 0)=\left(\frac{455}{1482}\right)^{\frac{1}{3}} \sec h^{\frac{4}{3}}\left[\frac{3}{2 \sqrt{29}} x\right] .
$$

For these parameters, the amplitude and velocity of the solitary wave are found as 0.30701 and $v=1.13495$, respectively. The experiment is run from $t=0$ to $t=$ 40 , and values of the invariants and error norms are shown in Table 3 . Table 3 shows that invariants are nearly constant over time.

It is noticeable from the table that the initial values of the invariants $I_{M}$ and $I_{E}$ change by less than $2.81 \times 10^{-5}$ for different values of $h$. Moreover, this study has found that error norms $L_{2}$ and $L_{\infty}$ are obtained small enough during the computer run. Therefore, the proposed method is sensibly conservative. The values of the error norms derived by our scheme are compared with those of schemes derived in $[7,9]$, as shown in Table 4 . This table clearly shows that the error norms obtained by the proposed method

\begin{tabular}{|c|c|c|c|c|}
\hline$h=0.5, \Delta t=0.1$ & $I_{M}$ & $I_{E}$ & $L_{2} \times 10^{-3}$ & $L_{\infty} \times 10^{-3}$ \\
\hline \multicolumn{5}{|l|}{$t$} \\
\hline 0 & 3.1329030 & 1.4338473 & 0 & 0 \\
\hline 10 & 3.1328960 & 1.4338400 & 0.84067 & 0.35644 \\
\hline 20 & 3.1328890 & 1.4338327 & 1.60312 & 0.63568 \\
\hline 30 & 3.1328819 & 1.4338254 & 2.34349 & 0.89170 \\
\hline 40 & 3.1328749 & 1.4338181 & 3.08384 & 1.15137 \\
\hline \multicolumn{5}{|l|}{$h=0.25, \Delta t=0.1$} \\
\hline \multicolumn{5}{|l|}{$t$} \\
\hline 0 & 3.1329030 & 1.4338473 & 0 & 0 \\
\hline 10 & 3.1328960 & 1.4338400 & 0.83723 & 0.35706 \\
\hline 20 & 3.1328890 & 1.4338327 & 1.59768 & 0.63291 \\
\hline 30 & 3.1328819 & 1.4338254 & 2.33605 & 0.89233 \\
\hline 40 & 3.1328749 & 1.4338181 & 3.07437 & 1.14864 \\
\hline
\end{tabular}

Table 3. The invariants and the error norms for single solitary wave for $p=4$ over the $-60 \leq x \leq 120$. 
Table 4. Comparison of error norms for $p=4$.

\begin{tabular}{|c|c|c|c|c|c|c|c|c|}
\hline \multirow[t]{2}{*}{$t=40}$, & \multirow[t]{2}{*}{$-60 \leq x \leq 120$} & \multirow[t]{2}{*}{$\Delta t=0.1$} & \multicolumn{3}{|c|}{$L_{2} \times 10^{-2}$} & \multicolumn{3}{|c|}{$L_{\infty} \times 10^{-3}$} \\
\hline & & & Present & {$[7]$} & [9] & Present & {$[7]$} & [9] \\
\hline & $h=0.5$ & & 0.30838 & 7.45173 & 0.44788 & 1.15137 & 27.87120 & 1.71122 \\
\hline & $h=0.25$ & & 0.30743 & 1.73066 & 0.47254 & 1.14864 & 6.47969 & 1.81252 \\
\hline
\end{tabular}

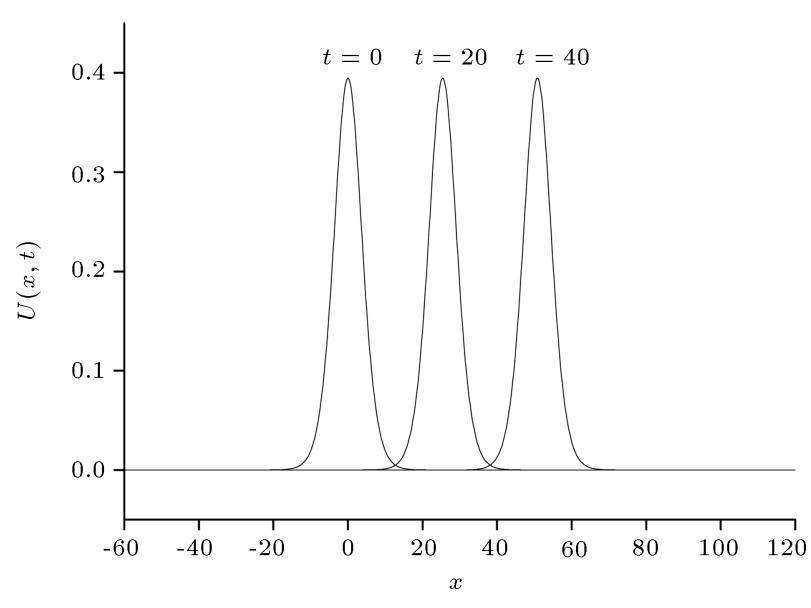

Figure 3. Motion of single solitary wave for $p=4$, $h=0.25$, and $\Delta t=0.1$ over the interval $[-60,120]$ at specified times.

are less than the others. For a visual representation, the simulations of single soliton for values of $h=$ $0.25, \Delta t=0.1$ at times $t=0,20$, and 40 are illustrated in Figure 3. According to this figure, the numerical scheme performs the motion of propagation of a single solitary wave, which acts on the right side at a nearly unchanged speed and conserves its amplitude and shape with the increasing amount of time. The amplitude is 0.674613 at $t=0$ and located at $x=0.0$, while it is 0.673988 at $t=40$ and located at $x=45.4$. A certain difference in amplitudes at times $t=0$ and $t=40$ is $6.25 \times 10^{-4}$ such that there is a minor difference between the amplitudes. Error distribution at time $t=40$ is depicted graphically in Figure 4 [38]. As is seen, the maximum number of errors occurs around the central position of the solitary wave.

\subsection{Case 3}

For the third numerical calculation, we have taken the parameter $p=8$ with values of $h=0.25,0.5$ and $\Delta t=$ 0.1 over the range $[-60,120]$. In this case, an exact solution and the initial condition for the problem are respectively found as follows:

$$
\begin{aligned}
& U(x, t)=\left(\frac{2475}{13802}\right)^{\frac{1}{7}} \sec h^{\frac{4}{7}}\left[\frac{7}{2 \sqrt{85}}\left(x-\frac{7225}{6901}\right) t\right] \\
& U(x, 0)=\left(\frac{2475}{13802}\right)^{\frac{1}{7}} \sec h^{\frac{4}{7}}\left[\frac{7}{2 \sqrt{85}} x\right] .
\end{aligned}
$$

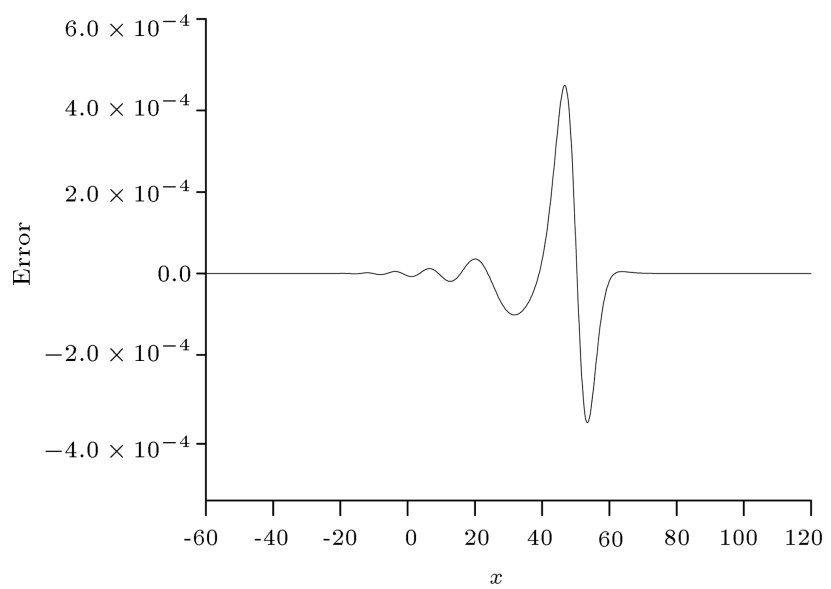

Figure 4. Errors for $p=4, h=0.25$, and $\Delta t=0.1$ at $t=40$.

For these parameters, the amplitude and velocity of a solitary wave are found to be 0.179321 and $v=$ 1.046949 , respectively. The algorithm is run at time $t=$ 40 to obtain the invariants and error norms in different time periods. The obtained results are tabulated in Table 5 . Table 5 shows that the invariants are nearly constant over time [30].

According to the table, the initial values of the invariants $I_{M}$ and $I_{E}$ change by less than $5 \times 10^{-5}$ for different values of $h$. Further, it is found that the error norms $L_{2}$ and $L_{\infty}$ are obtained as sufficiently small through the computer run. Thus, it can be argued that the proposed method is marginally conservative. Table 6 indicates a comparison of the values of the error norms derived by the proposed scheme and those derived by other schemes [7,9]. It is obviously seen from this table that the error norms obtained by the proposed method are smaller than those by other methods again [39]. The behaviors of solutions for values of $h=0.25, \Delta t=0.1$ at times $t=0,20$, and 40 are depicted in Figure 5. According to this figure, the solitary wave goes to the right at constant velocity and maintains its shape and amplitude. The amplitude is 0.782305 at $t=0$ and located at $x=0.0$, while it is 0.781552 at $t=40$ and located at $x=41.75$. The certain difference in amplitudes at times $t=0$ and $t=40$ is $7.53 \times 10^{-4}$; therefore, there is a minor difference between the amplitudes [34]. The error graph at $t=40$ is shown in Figure 6. It is observed that the maximum number of errors is associated with solitary waves and between $-4 \times 10^{-4}$ and $6 \times 10^{-4}[36]$. 
Table 5. The invariants and the error norms for single solitary wave for $p=8$ over the $-60 \leq x \leq 120$.

\begin{tabular}{|c|c|c|c|c|}
\hline$h=0.5, \Delta t=0.1$ & $I_{M}$ & $I_{E}$ & $L_{2} \times 10^{-3}$ & $L_{\infty} \times 10^{-3}$ \\
\hline \multicolumn{5}{|l|}{$t$} \\
\hline 0 & 4.8710424 & 2.3675820 & 0 & 0 \\
\hline 10 & 4.8710440 & 2.3675673 & 0.72292 & 0.30896 \\
\hline 20 & 4.8710430 & 2.3675526 & 1.50307 & 0.59229 \\
\hline 30 & 4.8710407 & 2.3675380 & 2.39050 & 0.90427 \\
\hline 40 & 4.8710374 & 2.3675234 & 3.40928 & 1.25518 \\
\hline \multicolumn{5}{|l|}{$h=0.25, \Delta t=0.1$} \\
\hline \multicolumn{5}{|l|}{$t$} \\
\hline 0 & 4.8710422 & 2.3675820 & 0 & 0 \\
\hline 10 & 4.8710454 & 2.3675673 & 0.71837 & 0.30641 \\
\hline 20 & 4.8710471 & 2.3675526 & 1.49461 & 0.58856 \\
\hline 30 & 4.8710482 & 2.3675380 & 2.37752 & 0.89891 \\
\hline 40 & 4.8710487 & 2.3675234 & 3.39111 & 1.12480 \\
\hline
\end{tabular}

Table 6. Comparison of error norms for $p=8$.

\begin{tabular}{ccccccccc}
\hline$t=40$, & $-60 \leq x \leq 120$, & $\Delta t=0.1$ & \multicolumn{3}{c}{$L_{2} \times 10^{-2}$} & & \multicolumn{3}{c}{$L_{\infty} \times 10^{-3}$} \\
& & Present & {$[7]$} & {$[9]$} & & Present & {$[7]$} & {$[9]$} \\
\cline { 2 - 6 } \cline { 4 - 7 } & 0.340928 & 8.03730 & 0.431841 & & 1.25518 & 29.5337 & 1.61891 \\
$h=0.5$ & & 0.339111 & 1.80583 & 0.46713 & & 1.24801 & 6.66740 & 1.75739 \\
\hline
\end{tabular}

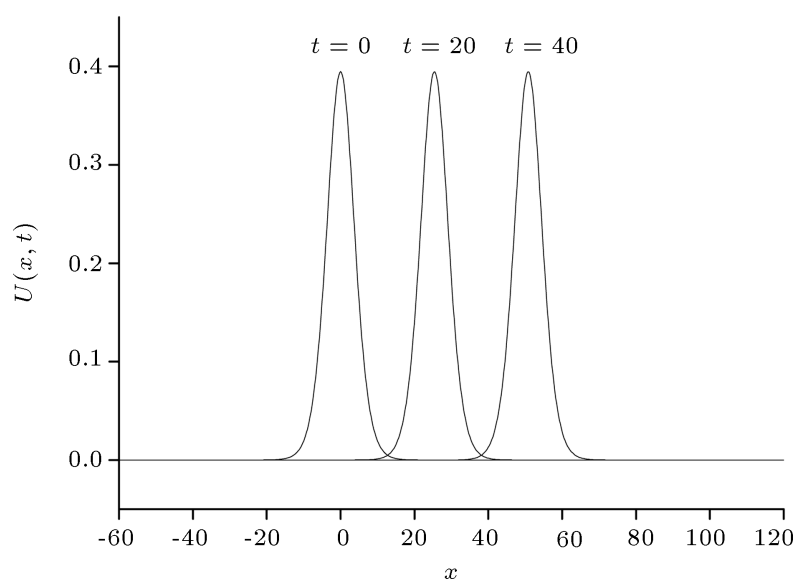

Figure 5. Motion of single solitary wave for $p=8$, $h=0.25$, and $\Delta t=0.1$ over the interval $[-60,120]$ at specified times.

\subsection{Case 4}

For the last numerical calculation, the problem with parameters $p=16, h=0.5,0.25$, and $\Delta t=0.1$ at the interval $[-60,120]$ is considered. In this case, since analytical solution of the problem is [9]:

$$
U(x, t)=\left(\frac{15827}{169386}\right)^{\frac{1}{15}} \sec h^{\frac{4}{15}}\left[\frac{15}{2 \sqrt{93}}\left(x-\frac{85849}{84693}\right) t\right]
$$

the initial condition for the problem is taken as follows:

$$
U(x, 0)=\left(\frac{15827}{169386}\right)^{\frac{1}{15}} \sec h^{\frac{4}{15}}\left[\frac{15}{2 \sqrt{93}} x\right]
$$

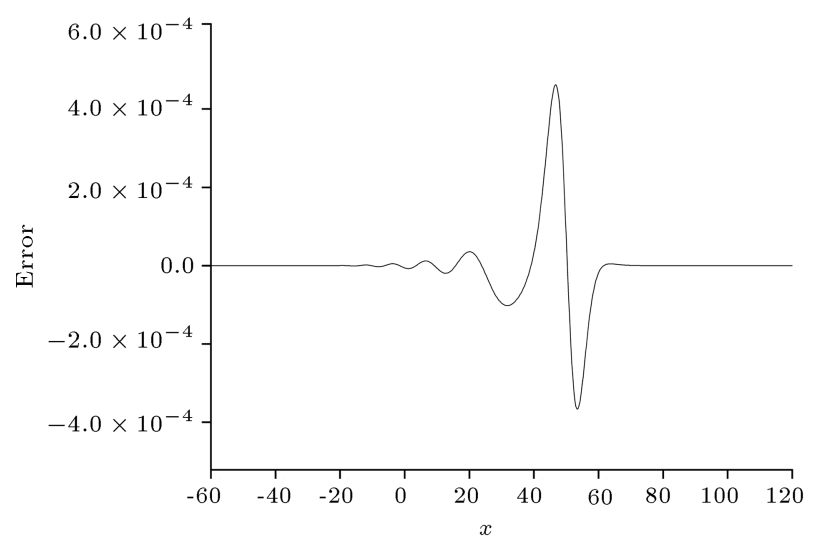

Figure 6. Errors for $p=8, h=0.25$, and $\Delta t=0.1$ at $t=40$.

For these parameters, the amplitude and velocity of the solitary wave are found as 0.093437 and $v=1.013649$, respectively. Error norms $L_{2}$ and $L_{\infty}$ and conserved quantities $I_{M}$ and $I_{E}$ are given in Table 7 up to time $t=40$. It is noticeably seen from Table 7 that invariants are nearly constant during the simulation, and the initial values of invariants $I_{M}$ change by less than $1 \times 10^{-2}$; however, the initial value of $I_{E}$ changes by less than $7 \times 10^{-5}$ for different values of $h$. Moreover, the error norms $L_{2}$ and $L_{\infty}$ are obtained small enough throughout the computer run. Therefore, the proposed method is sensibly conservative. Herein, the values of the error norms obtained by the present method are compared with those obtained by methods proposed in $[7,9]$, as shown in Table 8 . This table clearly shows 
Table 7. The invariants and the error norms for single solitary wave for $p=16$ over the $-60 \leq x \leq 120$.

\begin{tabular}{|c|c|c|c|c|}
\hline$h=0.5, \Delta t=0.1$ & $I_{M}$ & $I_{E}$ & $L_{2} \times 10^{-3}$ & $L_{\infty} \times 10^{-3}$ \\
\hline \multicolumn{5}{|l|}{$t$} \\
\hline 0 & 8.5743072 & 4.1877184 & 0 & 0 \\
\hline 10 & 8.5784605 & 4.1877007 & 1.10975 & 0.56601 \\
\hline 20 & 8.5817596 & 4.1876854 & 2.16628 & 0.71926 \\
\hline 30 & 8.5845639 & 4.1876709 & 3.10083 & 0.74798 \\
\hline 40 & 8.5868723 & 4.1876570 & 4.06967 & 1.01156 \\
\hline \multicolumn{5}{|l|}{$h=0.25, \Delta t=0.1$} \\
\hline \multicolumn{5}{|l|}{$t$} \\
\hline 0 & 8.5742501 & 4.1881116 & 0 & 0 \\
\hline 10 & 8.5789396 & 4.1880903 & 1.37957 & 0.63322 \\
\hline 20 & 8.5832196 & 4.1880718 & 2.76709 & 0.81657 \\
\hline 30 & 8.5872190 & 4.1880543 & 3.95618 & 0.86438 \\
\hline 40 & 8.5908517 & 4.1880374 & 5.09401 & 1.00763 \\
\hline
\end{tabular}

Table 8. Comparison of error norms for $p=16$.

\begin{tabular}{|c|c|c|c|c|c|c|c|}
\hline \multirow[t]{2}{*}{$t=40$} & \multirow[t]{2}{*}{$-60 \leq x \leq 120, \quad \Delta t=0.1$} & \multicolumn{3}{|c|}{$L_{2} \times 10^{-2}$} & \multicolumn{3}{|c|}{$L_{\infty} \times 10^{-3}$} \\
\hline & & Present & {$[7]$} & {$[9]$} & Present & {$[7]$} & {$[9]$} \\
\hline & $h=0.5$ & 0.406967 & 6.13044 & 0.357253 & 1.01156 & 22.5471 & 1.18759 \\
\hline & $h=0.25$ & 0.509401 & 1.37857 & 0.38438 & 1.00763 & 5.05919 & 1.30630 \\
\hline
\end{tabular}

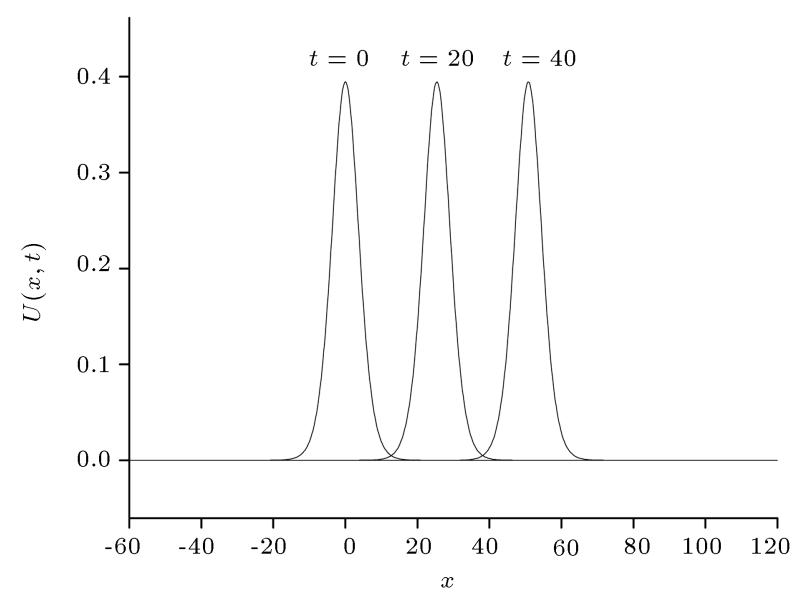

Figure 7. Motion of single solitary wave for $p=16$, $h=0.25$, and $\Delta t=0.1$ over the interval $[-60,120]$ at specified times.

that the error norms obtained by the proposed method are less than those by the others [39]. The profiles of the solitary wave for $h=0.25$ and $\Delta t=0.1$ at different time levels are shown in Figure 7 . Figure 7 displays that this method performs the motion of propagation of the solitary wave satisfactorily, which travels to the right at an invariable speed and nearly conserves its amplitude and shape with the increasing time [40]. The amplitude is 0.686098 at $t=0$ and located at $x=0$, while it is 0.685675 at $t=40$ and located at $x=43.250$. The absolute difference in amplitudes at times $t=0$ and $t=40$ is $4.2 \times 10^{-4}$ such that there is a minor difference between the amplitudes [34]. To

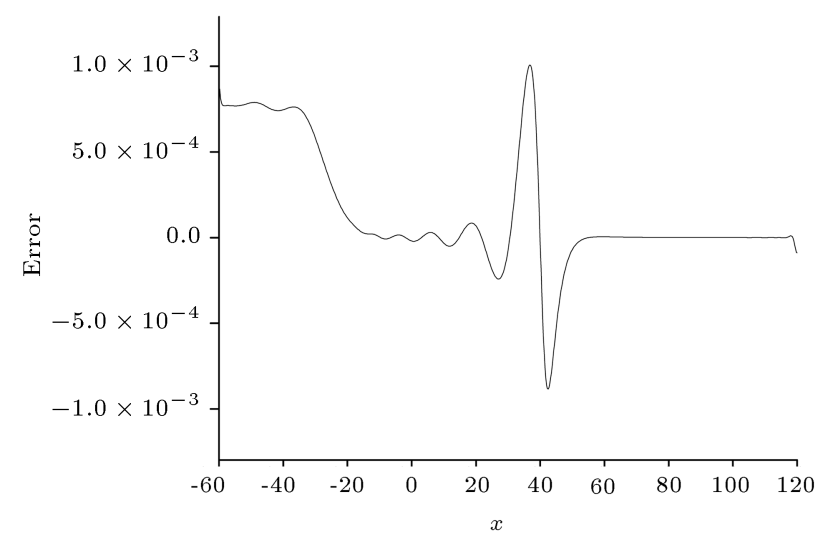

Figure 8. Errors for $p=16, h=0.25$, and $\Delta t=0.1$ at $t=40$.

represent the errors between the exact and numerical results over the problem region, error distributions are drawn for solitary waves at time $t=40$ in Figure 8 . It is indicated that the maximum number of errors is associated with the solitary waves and between $-1 \times 10^{-3}$ and $1 \times 10^{-3}[36]$.

\section{Conclusion}

This study successfully implemented the septic Bspline collocation method on the GR-RLW equation to analyze the motion of a single solitary wave whose analytical solution is known. To show how viable and accurate the numerical solutions of the test problems are, the error norms $L_{2}$ and $L_{\infty}$ and conserved 
quantities $I_{M}$ and $I_{E}$ were computed. The obtained numerical results indicated that the error norms were satisfactorily small and the conservation laws were marginally constant in all computer program runs. The proposed numerical scheme for the equation was found more accurate than the other previously mentioned schemes found in the literature. Stability analysis was carried out, and the linearized numerical scheme was obtained unconditionally stable. Therefore, it can be mentioned that the proposed numerical scheme is useful to obtain the numerical solutions of other important nonlinear problems in various fields.

\section{References}

1. Pan, X. and Zhang, L. "Numerical simulation for general Rosenau-RLW equation: An average linearized conservative scheme", Mathematical Problems in Engineering, 2012, Article ID 517818, 15 pages (2012).

2. Pan, X., Zheng, K., and Zhang, L. "Finite difference discretization of the Rosenau-RLW equation", Appl. Anal., 92, pp. 2578-2589 (2013).

3. Atouani, N. and Omrani, K. "Galerkin finite element method for the Rosenau-RLW equation", Computers and Mathematics with Applications, 66, pp. 289-303 (2013).

4. Yagmurlu, N.M., Karaagac, B., and Kutluay, S. "Numerical solutions of rosenau-RLW equation using Galerkin cubic B-spline finite element method", American Journal of Computational and Applied Mathematics, 7, pp. 1-10 (2017).

5. Wongsaijai, B. and Poochinapan, K. "A three-level average implicit finite difference scheme to solve equation obtained by coupling the Rosenau-KdV equation and the Rosenau-RLW equation", Applied Mathematics and Computation, 245, pp. 289-304 (2014).

6. Zuo, J., Zhang, Y.M., Zhang, T.D., et al. "A new conservative difference scheme for the general RosenauRLW equation", Bound. Value Probl., 2010, pp. 1-13 (2010).

7. Pan, X. and Zhang, L. "Numerical simulation for general Rosenau-RLW equation: An average linearized conservative scheme", Mathematical Problems in Engineering, 2012, Article ID 517818, 15 pages (2012).

8. Mittal, R.C. and Jain, R.K. "Numerical solution of general Rosenau-RLW, equations using quintic Bsplines collocation method", Commun. Numer. Anal., Article ID cna-00129, pp. 1-16 (2012).

9. Wongsaijai, B., Poochinapan, K., and Disyadej, T. “A compact finite difference method for solving the general Rosenau-RLW equation", IAENG International Journal of Applied Mathematics, 44, pp. 1-8 (2014).

10. Wang, H., Wang, J., and Li, S. "A new conservative nonlinear high order compact finite difference scheme for the general Rosenau-RLW equation", Bound. Value Probl., 2015, pp. 1-16 (2015).
11. Wang, H., Li, S., and Wang, J. "A conservative weighted finite difference scheme for the general Rosenau-RLW equation", Comp. Appl. Math., 36, pp. 63-78 (2017).

12. Arı, M. and Dereli, Y. "Numerical solutions of the general Rosenau-RLW equation using meshless kernel based method of lines", Journal of Physics: Conference Series, 766, pp. 1-6 (2016).

13. Cai, W., Sun, Y., and Wang, Y. "Variational discretizations for the generalized Rosenau type equations", Appl. Math.Comput., 271, pp. 860-873 (2015).

14. Korteweg, D.J. and de Vries, G. "On the change of form of long waves advancing in a rectangular canal, and on a new type of long stationary wave", Philosophical Magazine, 39, pp. 422-443 (1895).

15. Raslan, K.R., El-Danaf, Talaat S., and Ali, Khalid K. "Application of linear combination between cubic B-spline collocation methods with different basis for solving the KdV equation", Computational Methods for Differential Equations, 4, pp. 191-204 (2016).

16. Raslan, K.R., El-Danaf, Talaat S., and Ali, Khalid K. "Collocation method with quintic b-spline method for solving hirota-satsuma coupled KDV equation", International Journal of Applied Mathematical Research, 5, pp. 123-131 (2016).

17. Raslan, K.R., El-Danaf, Talaat, S., and Ali, Khalid, K. "New numerical treatment for solving the KDV equation", Journal of Abstract and Computational Mathematics, 2, pp. 1-12 (2017).

18. Peregrine, D.H. "Calculations of the development of an undular bore", J. Fluid Mech., 25, pp. 321-330 (1966).

19. El-Danaf, Talaat S., Raslan, K.R., and Ali, Khalid K. "New numerical treatment for the generalized regularized long wave equation based on finite difference scheme", Int. J. of S. Comp. and Eng. (IJSCE)', 4, pp. 16-24 (2014).

20. El-Danaf, Talaat, S., Raslan, K.R., and Ali, Khalid K. "Collocation method with cubic B-splines for solving the GRLW equation", Int. J. of Num. Meth. and Appl., 15, pp. 39-59 (2016).

21. Raslan, K.R., El-Danaf, Talaat S., and Ali, Khalid K. "New exact solution of coupled generalized regularized long wave equation", Journal of the Egyptian Mathematical Society, 25, pp. 400-405 (2017).

22. Raslan, K.R., Ali, Khalid K., and Shallal, Muhannad A. "Solving the space-time fractional RLW and MRLW equations using modified extended tanh method with the Riccati equation", British Journal of Mathematics \& Computer Science, 21, pp. 1-15 (2017).

23. El-Danaf, Talaat S., Raslan, K.R., and Ali, Khalid K. "Non-polynomial spline method for solving the generalized regularized long wave equation", Communication in Mathematical Modeling and Applications, 2, pp. 1-17 (2017).

24. Karakoc, S.B.G., Ucar, Y., and Yagmurlu, N.M. "Numerical solutions of the MRLW equation by cubic Bspline galerkin finite element method", Kuwait Journal of Science, 42, pp. 141-159, (2015). 
25. Rouzegar, J. and Sharifpoor, R. Abdoli. "A finite element formulation for bending analysis of isotropic and orthotropic plates based on two-variable refined plate theory", Scientia Iranica, B, 22, pp. 196-207 (2015).

26. Pan, X. and Zhang, L. "A new finite difference scheme for the Rosenau-Burgers equation", Applied Mathematics and Computation, 218, pp. 8917-8924 (2012).

27. Rosenau, P. "A quasi-continuous description of a nonlinear transmission line", Phys. Scripta., 34, pp. 827-829 (1986).

28. Rosenau, P. "Dynamics of dense discrete systems", Progr. Theory. Phys., 79, pp. 1028-1042 (1988).

29. Park, M.A. "On the Rosenau equation", Math. Appl. Comput., 9, pp. 145-152 (1990).

30. Karakoc, S.B.G. and Zeybek, H. "Solitary-wave solutions of the GRLW equation using septic B-spline collocation method", Applied Mathematics and Computation, 289, pp. 159-171 (2016).

31. Prenter, P.M., Splines and Variational Methods, John Wiley \& Sons, New York, NY, USA (1975).

32. Esen, A. "A lumped Galerkin method for the numerical solution of the modified equal-width wave equation using quadratic B-splines", International Journal of Computer Mathematics, 83, pp. 449-459 (2006).

33. Saka, B., Şahin, A., and Dă̆, I. "B-spline collocation algorithms for numerical solution of the RLW equation", Numer. Methods Partial Differential Eq., 27, pp. 581-607 (2011).

34. Geyikli, T. and Karakoc, S.B.G. "Septic B-spline collocation method for the numerical solution of the modified equal width wave equation", Applied Mathematics, 2, pp. 739-749 (2011).

35. Ak, T., Dhawan, S., Karakoc, S.B.G., et al. "Numerical study of Rosenau-KdV equation using finite element method based on collocation approach", Mathematical Modelling and Analysis, 22, pp. 373-383 (2017).

36. Karakoc, S.B.G., Yagmurlu, N.M., and Ucar, Y. "A numerical approximation to solution of the modified regularized long wave (MRLW) equation using quintic B-splines", Bound. Value Probl., 27, pp. 1-17 (2013).

37. Ak, T., Karakoc, S.B.G., and Biswas, A. "Application of Petrov-Galerkin finite element method to shallow water waves model: Modified Korteweg-de Vries equation", Scientia Iranica B., 24, pp. 1148-1159 (2017).

38. Hassan, H.N. "An accurate numerical solution for the modified equal width wave equation using the Fourier pseudo-spectral method", Journal of Applied Mathematics and Physics, 4, pp. 1054-1067 (2016).

39. Essa, Y.M. Abo "Multigrid method for the numerical solution of the modified equal width wave equation", Applied Mathematics, 7, pp. 1140-1147 (2016).

40. Karakoc, S.B.G. and Geyikli, T. "Numerical solution of the modified equal width wave equation", International Journal of Differential Equations, 2012, pp. 115 (2012).

\section{Biography}

Seydi Battal Gazi Karakoc is working as an Associate Professor at the Department of Mathematics, Faculty of Science and Art, University of Nevsehir Haci Bektas Veli, Turkey. He earned his BSc from Selcuk University. Then, he earned his MSc and PhD degrees in Applied Mathematics from Inonu University. His research interest areas include numerical analysis, computational methods, mathematical modeling of wave equations, and partial differential equations. 